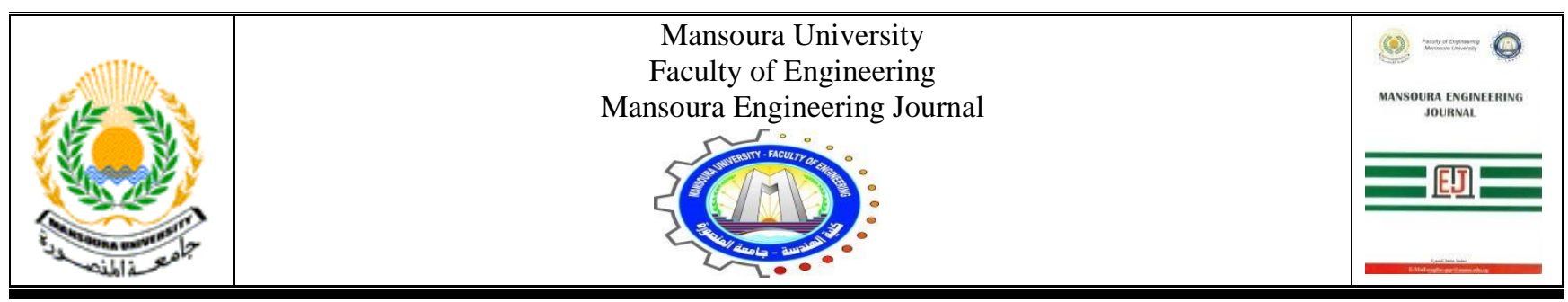

\title{
Evaluation of Common Traffic Operation Softwares on the Basis of Relevancy to Roads Intersections
}

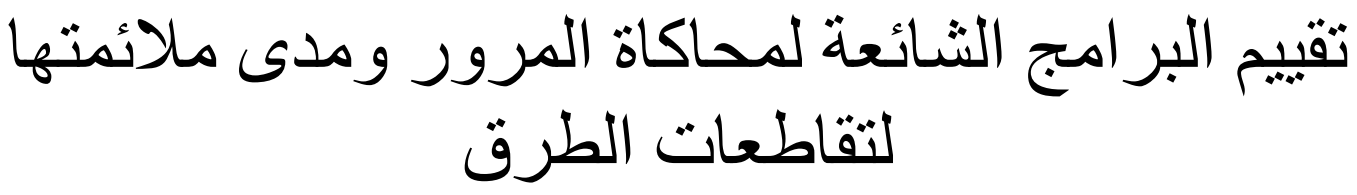

Ahmed Mohamady Abd-Allah, Hassan Darwish Hassanin, Manal Abd-Allah Ahmed and Abdelrahman Baz Abdelsemii

KEYWORDS:
Control delay time,
HCM, Synchro 7.0,
HCS2000, SIDRE
INTERSECTION 5.1
and Calibration

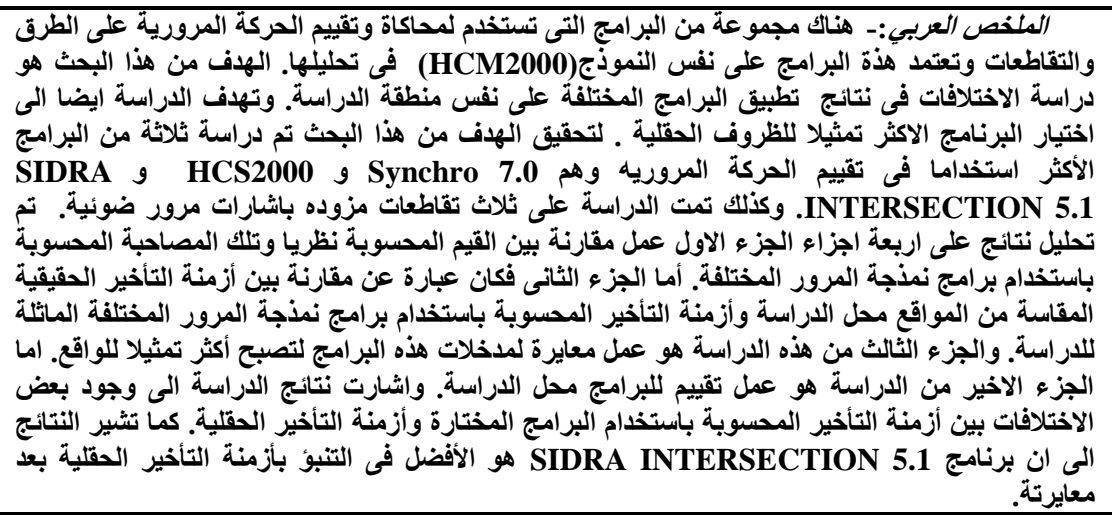

this study; they included SYNCHRO 7, HCS2000 and SIDRA INTERSECTION 5.1. The main objective of this study is to investigate the main causes of these differences as well as defining one of these programs to be the most representative of traffic movement on intersections and develop it. To achieve this goal, three signalized intersections were selected. The analysis of results included four main subjects; the first subject included a comparison between different investigated packages outputs and HCM methodology. The second subject included a comparison between delay times measured from the field and the corresponding delay times determined using the selected programs. The third subject included calibrating the selected software programs. Finally, evaluation to real delay times at various study cases and their corresponding values determined using the selected software packages are conducted. It can be concluded that there are significant differences between the field measured delay times and the corresponding calculated delay

\footnotetext{
Received: 6 September, 2015 - revised: 15 May, 2016 - accepted: 26 Dr. Ahmed Mohamady Abd-Allah, Assoc. Prof. Of Highways and Airport Engineering, Faculty of Engineering, Zagazig University

Dr. Hassan Darwish Hassanin, Lecturer of Highways and Airports Engineering, Faculty of Engineering, Zagazig University

Dr. Manal Abd-Allah Ahmed, Lecturer of Highways and Airports Engineering, Faculty of Engineering, Zagazig University

Eng. Abdelrahman Baz Abdelsemii, B. Sc, Civil Engineering, Zagazig University, 2010.
} 
times using the selected software programs. It is concluded that SIDRA INTERSECTION 5.1 software after its calibration is considered the best software for predicting the field delay times at signalized intersection.

\section{INTRODUCTION}

$\mathrm{I}$ $\mathrm{n}$ recent years, there are many traffic operation software that used to simulate and evaluate traffic operation of roads and intersections. This traffic software is available to a lot of specialists in this area and they give variable results for the same locations. So it is vital to compare between the most common traffic softwares used in Egypt to find out the reasons of the different results and to determine the appropriate conditions for applying or using any of them. Recent advances in computer technology and traffic flow theory have led to the widespread creation and use of traffic simulation models of traffic engineers and transportation planners involved in the planning, operations, and design of transportation facilities [1]. Growing competition in many industries has resulted in a greater emphasis on developing and using automated manufacturing systems to improve productivity and to reduce costs. Due to the complexity and dynamic behavior of such systems, simulation modeling is becoming one of the most popular methods of facilitating their design and assessing operating strategies [2]. A reliable description of traffic flow is a nontrivial problem. A lot of models have been proposed so far, unfortunately, none of them can be considered as an ideal or, at least, universal one [3]. Computer simulation is a widely used method in research on traffic modeling, planning and development of traffic networks and systems. Vehicular traffic systems are of growing concern and interest globally and modeling arbitrarily complex traffic systems is a hard problem [4].

Previous studies compared between a lots of traffic software that are used to study the traffic movement. This study will investigate three traffic softwares; Synchro7.0, Highway Capacity Software (HCS2000) and SIDRA INTERSECTION 5.1.

Omari and Madhar M. Ta'amn compared between HCS and SIDRA. The results of the analysis showed that, for low delay ranges, HCS tends to slightly over-estimate control delay; while SIDRA has a predicted control delay that is in good agreement with the field data. At higher delay levels, HCS has noticeable scattered predictions as compared to field data with more tendencies to over-estimate, while SIDRA provides less scattering than HCS. It was found that the two models can be improved significantly by calibrating bus Passenger Car Equivalent (PCE) factor or the basic saturation flow rate. However, the best improvement could be obtained by calibrating both the basic saturation flow rate and bus PCE factor, simultaneously. It can be concluded that the two softwares can be used for traffic analysis in Jordanian conditions after calibrating both the saturation flow rate and bus PCE factor. However, SIDRA showed a better performance than HCS [5].

Abeer K. Jameel found that the method of statically comparison has been used in many research to validate the theoretical model results with the actual value. The results of the analysis showed that the Highway Capacity Manual (HCM) model using HCS and SIDRA INTERSECTION4 with HCM model gives a predicted control delay that is best agreement with the field data is SIDRA model. This study showed that traffic softwares, which are being used in the developed countries, should not be used in Iraq or other developing countries before calibrating their parameters that are believed to be different from those in developed countries such as the ones related to driver behavior [6].

The main objective of this study is to investigate the main causes of these differences as well as defining one of these software packages to be the most representative of traffic movement on the road links and intersections and develop it.

This study consists of four parts; the first part is this introduction that presents a very bravely literature review. The second part is the methodology that presents the main steps that followed to achieve the objectives of the study. The third part includes the analysis of results and discussion. The last part is the study's conclusion that summarizes the final findings of the study.

\section{METHODOLOGY}

Extensive experimental program was designed and implemented to achieve study objectives. The experimental program composed of both office and field work as shown in Figure (1).

\section{A. The Study Program}

Figure (1) shows that experimental program is composed of office work and field work. The office work began with making thoroughly reviewing about the most famous software packages; SYNCHRO 7.0, HCS2000, and SIDRA INTERSECTION 5.1. The review also included defining of different prevailing field conditions of both roads intersections and links at the study area. These prevailing conditions were used as a base for selection of the investigated roads intersections. The chosen study cases included three major signalized intersections in Zagazig city. They included Alqawmia, Alaboor and Sawarese intersections.

The review was extended to define different data required to achieve the objectives of this study. A search about the different data affecting on intersection capacity and delay time calculations was carried out. It was found that these data were related to geometrics, traffic, and control types.

The field work stage was started after defining the required data. The required data were collected by trained team. The collected data were then used to make a complete analysis that included making the validation and calibration of the chosen software packages. A detailed comparison was carried out between delay time measured from field data and those 
resulted from applying the selected software packages. Collection of the research conclusions and recommendations was carried out based on salary analysis of the data collected.

\section{B. Data Collection}

The collected data for traffic analysis was grouped as geometric data, traffic data, control data, and field delay time. Groups of students from the last level of Faculty of Engineering, Zagazig University, were carefully trained to be the team who collected the required field different data according to specified rules as described in the following sections.

1) Geometric Data:

The required intersections geometric data were that providing complete knowledge about capacity and delay time. These data included general data and layout data. General and site information data were composed of intersection name, area type, date, and year of survey, number of intersection legs, and post speed for all intersection approaches. Layout data included approach direction, pavement type, and number of lanes for all intersection legs, lane width, lane purpose, parking maneuvers, storage length, median width, shoulder width, and sidewalk width.

2) Traffic Data:

Traffic volumes were counted manually through field survey at all lane groups and aggregated over 15-minute time intervals. The required data classified to general data and volume data. General and site information data composed of intersection name, area type, date, and year of survey. Volume data included traffic volume, heavy vehicle percent, peak hour factor, arrival type, pedestrian volume, and bicycle volume. The manual survey was defined such that it covered peak and off-peak hours. Count interval from 7.00 AM to 11.00 AM was selected to cover Morning peak and from 1.00 PM to 5.00 PM was selected to cover evening peak. Traffic volumes were recorded on three days; Sunday, Tuesday and Thursday per week in June 2013 for each approach of the selected study cases. The passenger car equivalent for the heavy vehicles was calculated and equal 1.5.

3) Control Data:

Intersection control data included control type, signal phasing, timing plans, signing, and pavement markings.

4) Field Delay Time Measuring Procedure:

Field delay time was counted at three signalized intersections. The survey was conducted on both peak and off-peak hours. Minimum survey time was one hour per day for a set of representative days. Thirteen hours were counted at the selected intersections; seven hours at Alqawmia intersection, three hours at Alaboor intersection, and three hours at Sawarss intersection. The survey was conducted by using the HCM2000 worksheet. That classified the required data to three groups; general site data, initial data, queuing data. General site information data included intersection name, area type, date, and year of survey. Initial data included number of lanes of counted approaches, free flow speed, count interval, number of stopped vehicle during count period, and total number of vehicles arriving during the survey period. During collecting the field data, it was found that it is very difficult to determine the number of vehicles in queue at each count interval. So, video recording was used for collecting data for the intersection approaches to determine the sum of vehicles in queue.

\section{RESULTS AND ANALYSIS}

This section presents the analysis of the study results through four sections as follow.

\section{A. Delay Time Comparison}

This section presents the comparison between the delay times calculated theoretically and the corresponding delay times determined using SYNCHRO 7, HCS2000, and SIDRA INTERSECTION 5.1 software packages at signalized intersections.

1) Comparison between Delay Times Determined by SYNCHRO 7 Software and Theoretical Delay Times:

Figure (2) shows the relationship between the theoretical calculated delay times and the calculated delay times using SYNCHRO 7 software for Alqawmiaa, Alaboor and Sawarss intersections. The figure presents the comparison for both peaks and off-peaks hours at all intersections approaches. It shows that there are slight differences between delay times calculated using SYNCHRO 7 Software and the corresponding calculated delay times using HCM2000. At the level of intersection as whole, SYNCHRO 7 delay times are slightly greater than the theoretical calculated delay times by $3.64 \%$ in peak hours. This increase in delay time decreases to $1.70 \%$ in the case of off-peak hours while for intersections approaches the theoretical delay times are greater than SYNCHRO 7 delay times by values range between $0.48 \%$ and $5.17 \%$. As previously shown, it can be deduced that the recorded difference are very small. This can be explained by SYNCHRO 7 Software follow HCM2000 in all analysis steps.

2) Comparison between Delay Times Determined by HCS2000 Software and Theoretical Delay Times:

Figure (3) shows that there are linear relationships between the theoretical calculated delay times $(\mathrm{X})$ and the delay times calculated using HCS2000 software (y) for the investigated signalized intersections. The relationship can be described by the following Equation:

$$
\mathrm{y}=1.03 \mathrm{X} \text { and } \mathrm{R} 2=99.87 \%
$$

The figure also presents the comparison between the theoretical calculated delay times and the delay times calculated using HCS2000 Software for both peak and offpeak hours at all intersections approaches. The figure shows that there are slight differences between delay times calculated using HCS2000 Software and the corresponding delay times calculating using HCM2000. At the level of intersection, HCS2000 delay times are greater than the 
calculated delay times by $(3.14 \%$ and $0.58 \%)$ in peak and off-peak hours respectively. For the intersections approaches, the delay times calculated using HCS2000 are greater than the theoretical calculated delay times with values range from $0.48 \%$ to $5.85 \%$. It can be deduced that the recorded difference is very small.

3) Comparison between Delay Times Determined by SIDRA Intersection 5.1 Software and Theoretical Delay Times:

Figure (4) shows the relationship between the theoretical calculated delay times and the delay times calculated using SIDRA INTERSECTION 5.1 Software for studied signalized intersections ( Alqawmiaa, Alaboor, and Sawarss intersections). The figure presents the comparison for both peaks and off peaks hours at all intersections approaches. It illustrates that there are noticeable differences between delay times calculated using SIDRA INTERSECTION 5.1 Software and the corresponding delay times calculating using HCM2000. At the level of intersection as a whole, the calculated delay times are greater than SIDRA INTERSECTION 5.1 delay times by values $(17.56 \%$ and $1.80 \%)$ for peak hours and off peak hours. At the level of intersections approaches, the theoretical delay times are lower than the corresponding delay times determined using SIDRA 5.1 with values range between $0.24 \%$ and $38.40 \%$. This may be due to the neglecting of area adjusted factor by SIDRA INTERSECTION 5.1 which led to increasing of adjusted saturation flow, consequently decreasing calculated delay times.

\section{B Softwares Validation}

This section presents the validation process that carried out to the different investigated software packages. The validation of any software is conducted through making comparison between field measurement delay times and the corresponding delay times determined using this software. Field delay times were surveyed through thirteen hours for the three investigated signalized intersections.

1) Synchro 7 Software Validation:

Figure (5) shows the relationship between the field measurement delay times and the corresponding delay times calculated by using SYNCHRO 7 Software at all signalized intersections approaches. This relationship was considered a strong linear relationship where: $y=1.2559$ $\mathrm{X}$ and $\mathrm{R} 2$ equals to $72.24 \%$. The figure also shows that delay times determined by using SYNCHRO 7 are greater than the corresponding field measurement delay times with values ranging from $1.23 \%$ to $40.26 \%$. These differences are considered significant differences. SYNCHRO 7 uses default base saturation flow rate 1900 pcphgpl. Adjusting this saturation flow by different factors which leads to decreasing lane capacity, consequently increasing calculated delay times.

2) HCS2000 Software Validation:

The relationships that represent the comparison between the field measurement delay times and the corresponding delay times calculated by using HCS2000 Software at the investigated signalized intersections approaches shown in Figure (6). This relationships was strong linear relationships where: $\mathrm{y}=1.3253 \mathrm{X}$ and $\mathrm{R} 2$ equals to $70.64 \%$. The figure also shows that delay times determined by using HCS2000 Software was greater than the corresponding field measurement delay times with values ranging from $2.30 \%$ to $54.26 \%$. These differences were considered significant differences

3) SIDRA Intersection 5.1 Software Validation:

Figure (7) shows the relationship between the field measurement delay times and the corresponding delay times determined by using SIDRA INTERSECTION 5.1 Software at signalized intersections approaches. The relation can be described by the following Equation:

$$
\mathrm{y}=1.145 \mathrm{X} \text { and } \mathrm{R} 2=75.46 \% \text {. }
$$

This relationship was considered a strong linear relationship. The figure illustrated that delay times determined by using SIDRA INTERSECTION 5.1 were greater than the corresponding field measurement delay times with values ranging from $2.00 \%$ to $32.92 \%$. These differences were considered significant differences. SIDRA INTERSECTION 5.1 used default base saturation flow rate 1950 pcphgpl. It was neglected the area adjusted factor that led to decreasing its delay times.

\section{Softwares Calibration}

The analysis of results at section 5.2 showed that delay times determined by using different software packages ware usually greater than the corresponding real delay times measured from field. The study aims to adjust some inputs of the investigated software programs as a trial to decrease the difference between their output delay times and the corresponding delay times measured from field. This what the study means by software calibration. The inputs of the different software packages are about twelve factors. These factors effect on saturation flow rate which was used to determine lane capacity. The most important factors among the previous factors are: (a) base saturation flow rate, (b) lane width adjusted factor, (c) heave vehicle adjusted factor, and (d) area adjusted factor. The study suggested different values of base saturation flow $(1900,2000,2100$, and 2200 pcphgpl) for all software programs. The study also neglected area adjusted factor. All these suggestions ware to be applied within the inputs of the different software programs with the fixation of all other inputs as a trial to increase lane capacity and consequently decreasing the determined delay times.

1) Figures from (8 to 11) show the linear relationships and the correlation factors between the field measurement delay times and the corresponding adjusted delay times determined by using SYNCHRO 7 Software after neglecting area adjusted factor and considering different values of base saturation flow. It is note that using 1900 $\mathrm{pc} / \mathrm{hrg} / \mathrm{lane}$ base saturation flow and neglecting area adjusted factor (Figure 8) leads to increasing R2 from $74.6 \%$ to $72.24 \%$. This may be due to neglecting area 
adjusted factor .The Figures $(9,10$, and 11) illustrate that R2 increases with the increasing of base saturation flow. This increasing is due to the increasing lane capacity and consequently decreasing the determined delay times. The nearest determined delay times using SYNCHRO 7 Software are at 2200 pcphgpl as a base saturation flow with neglecting area factor (Figure 11). So, the study recommends using 2200 pcphgpl as a base saturation flow with neglecting area factor as a calibration to SYNCHRO 7 Software.

2) Figures from (12 to 15) show the linear relationships and the correlation factors between the field measurement delay times and the corresponding adjusted delay times determined by using HCS2000 Software after neglecting area adjusted factor and considering different values of base saturation flow. Figure (12) presents this relationship using 1900 pcphgpl base saturation flow and neglecting area adjusted factor. This figure illustrates that R2 increases from $73.89 \%$ to $70.64 \%$. This increasing may be due to neglecting area adjusted factor. The Figures from (13 to 15) illustrate that R2 increases with the increasing of base saturation flow. This increasing is due to the increasing lane capacity and consequently decreasing the determined delay times. The beast determined delay times using HCS2000 Software are at 2200 pcphgpl as a base saturation flow with neglecting area factor (Figure 15). So, the study recommends using 2200 pcphgpl as a base saturation flow with neglecting area factor as a calibration to HCS2000 Software.

3) SIDRA Intersection 5.1 Software Calibration:

Figures from (16 to 19) show the linear relationships and the correlation factors between the field measurement delay times and the corresponding adjusted delay times determined by using SIDRA INTERSECTION 5.1 Software at different suggested values of base saturation with neglecting area adjusted factor. The Figures $(16,17$, 18 , and 19) illustrate that R2 increases with the increasing of base saturation flow and neglecting area adjusted factor. This increasing is due to the increasing lane capacity and consequently decreasing the determined delay times. The nearest determined delay times using SIDRA INTERSECTION 5.1 Software are at 2200 pcphgpl as a base saturation flow with neglecting area factor (Figure 19). So, the study recommends using 2200 pcphgpl as a base saturation flow with neglecting area factor as a calibration to SIDRA INTERSECTION 5.1 Software.

\section{Softwares Evaluation}

The objective of this section is to select the more representative software to the field delay time measurements. To achieve this objective, a comparison between the field delay measurements and the corresponding delay times determined by using the different software programs at 2200 pcphgpl base saturation flow rate with area factor equal to 1.00. Figure (20) shows this comparison. The figure shows that the stronger relationship is between field delay time measurements and delay times calculated using SIDRA INTERSECTION 5.1 after calibration. The equation represents this relation is $\mathrm{y}=1.0497 \mathrm{X}$. It achieves the highest coefficient of correlation $(\mathrm{R} 2=0.7811)$. The delay times determined by using other software programs after calibration are closer to corresponding field delay time measurements than those determined by using SYNCHRO 7, HCS2000 before calibration. So, the study recommends using SIDRA INTERSECTION 5.1 Software after calibration to predict field delay times for signalized intersections.

\section{CONCLUSION}

Based on the results of this study, the following conclusions can conclude that:

1. By comparison between softwares delay times and the corresponding delay times calculated theoretically using HCM2000;

(a) Insignificant differences were noticed between delay times calculated using both of SYNCHRO 7.0 and HCS2000 Softwares and those calculated by HCM methodology. SIDRA INTERSECTION 5.1 Software has significant differences in its calculated delay times.

(b) HCS2000 Software can calculate delay times at signalized intersections with error $1.86 \%$ and $\mathrm{R} 2=99.87 \%$ comparing with HCM2000 Delay times.

(c) SYNCHRO 7 Software can calculate delay times at signalized intersections with average error $2.67 \%$ and $\mathrm{R} 2=$ 99.93\% comparing with HCM2000 Delay times.

(d) SIDRA 5.1 Software can calculate delay times at signalized intersections with average error $9.68 \%$ and R2 $=$ $69.20 \%$ comparing with HCM2000 Delay times.

(e) HCS2000 and SYNCHRO 7 Softwares are the best softwares for calculating the delay times at intersections compared with the delay times calculated theoretically by using HCM2000.

2. SYNCHRO 7 software is considered the most reliable Software regarding data entry and identification of analysis outputs.

3. It was found that there are significant differences between the field measured delay times and the corresponding delay times calculated using SYNCHRO 7.0, HCS2000, and SIDRA INTERSECTION 5.1 Softwares.

4. The linear relationship between the field delay times and different Softwares delay times was predicted. These relationships were as follows:

$$
\begin{aligned}
& \mathrm{y}=1.2550 X \text { and R2 }=72.24 \% \quad \text { (SYNCHRO). } \\
& \mathrm{y}=1.3253 \mathrm{X} \text { and R2 }=70.64 \% \quad \text { (HCS). } \\
& \mathrm{y}=1.1450 \mathrm{X} \text { and } \mathrm{R} 2=75.46 \% \quad(\text { SIDRA). }
\end{aligned}
$$

5. The calculated delay times using different softwares became more matching with field measured delay times after calibration but the differences were still significant.

6 . The advanced equations representing the relation between field delay times and the calibrated softwares delay times are as follows:

$$
\begin{array}{ll}
\mathrm{y}=1.0064 X \text { and } \mathrm{R} 2=76.40 \% & (\text { SYNCHRO }) \\
\mathrm{y}=1.0375 \mathrm{X} \text { and } \mathrm{R} 2=74.84 \% & (\text { HCS }) . \\
\mathrm{y}=1.00497 \mathrm{X} \text { and } \mathrm{R} 2=78.11 \% & (\text { SIDRA })
\end{array}
$$


7. SIDRA INTERSECTION 5.1 Software is considered the best software for prediction of the field delay times at signalized intersections after the calibration process.

\section{REFERENCES}

[1] Institute of Transportation Engineers California Border Section Highway Capacity Task Force, "A Report on The Use of Traffic Simulation Models in the San Diego Region", March 17, 2004.

[2] Rajesh Verma, Ashu Gupta and Kawaljeet Singh (A Critical Evaluation And Comparison Of Four Manufacturing Simulation Softwares) Kathmandu University Journal Of Science, Engineering And Technolgy Vol. 6, No. I, January, 2009, Pp 104- 120.

[3] Michal Maciejewski (A Comparison of Microscopic Traffic Flow Simulation Systems for an Urban Area) (volume 5,2010).

[4] G. Kotusevski and K.A. Hawick (A Review of Traffic Simulation Software), July 23, 2009.
[5] Bashar H. Al-Omari and Madhar M. Ta'amneh (Validating HCS and Sidra Software for Estimating Delay at Signalized Intersections in Jordan), Jordan Journal of Civil Engineering, Volume 1, No. 4, pp. 375392, 2007.

[6] Abeer K. Jameel "Estimating Delay Time at Palestine Street Intersections in Baghdad City Using HCM and Sidra Models", 2011.

[7] Kenneth J. Petraglia (Field Validation of Moe's for Signalized Intersection Analysis),

[8] Shivam Gupta CE 683 Traffic Engineering (Delay at Signalized Intersection) Indian Institute of Technology, Kanpur.

[9] Transportation Research Board (TRB), Highway Capacity Manual. National Research Council. Washington, D.C., 2000.

[10]Trafficware. Synchro Version 7. Berkeley, California, 1998.

[11]Akçelik, R., "SIDRA5.1 INTERSECTION User Guide", Victoria, Australia, 2009

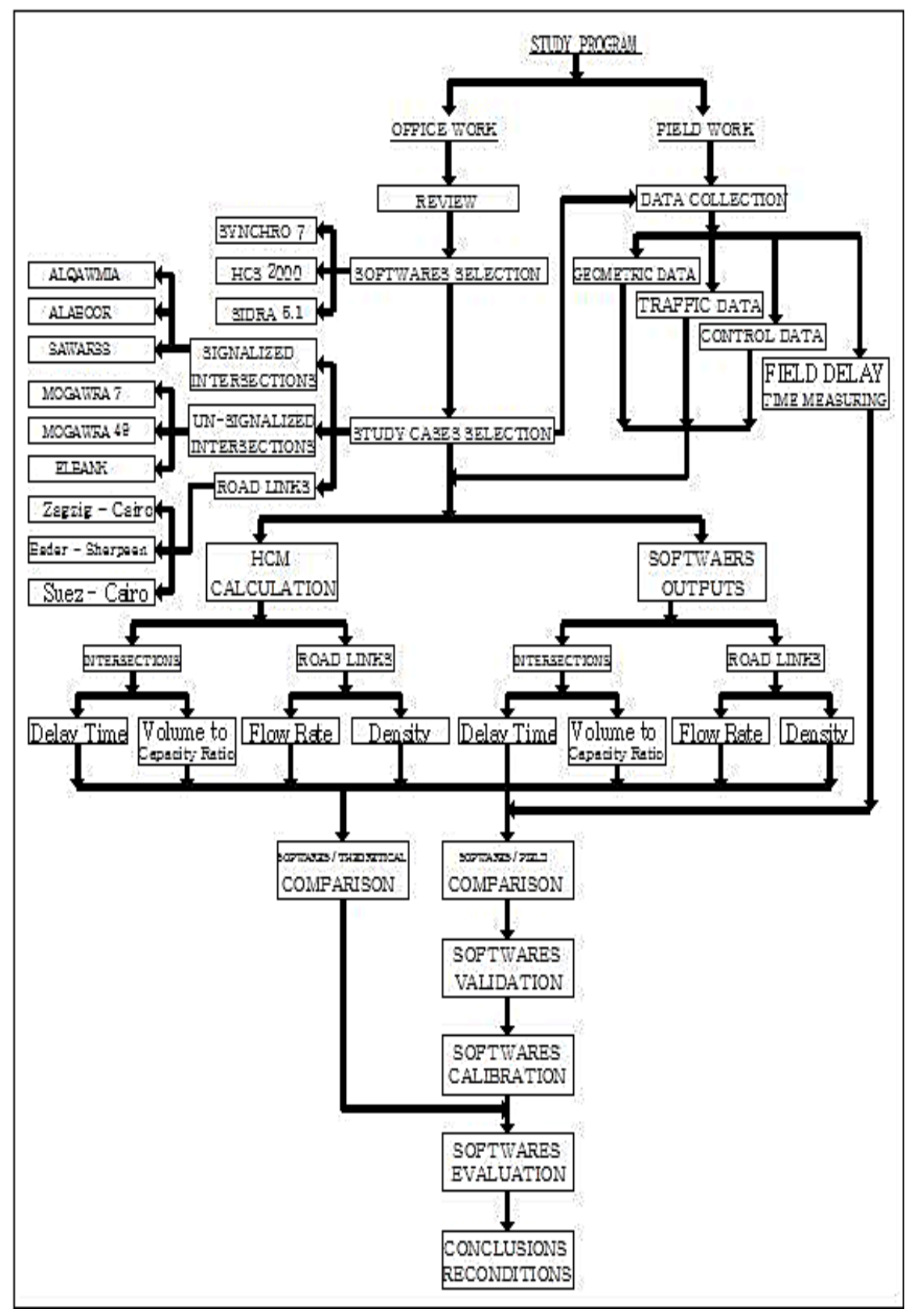

Figure (1): Flow Chart Showing Experimental Program 
C: 22 MANSOURA ENGINEERING JOURNAL, (MEJ), VOL. 41, ISSUE 2, JUNE 2016

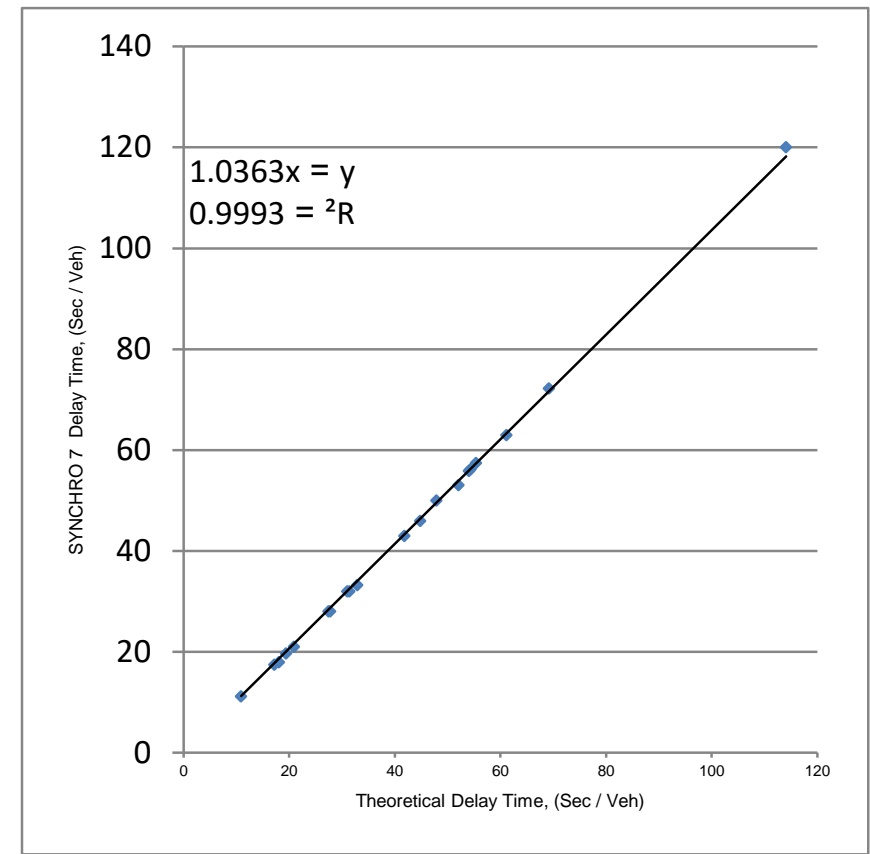

Figure (2): Relationship between Delay Times Calculated Theoretically and Delay Times Determined Using SYNCHRO 7 Software at Signalized Intersections.

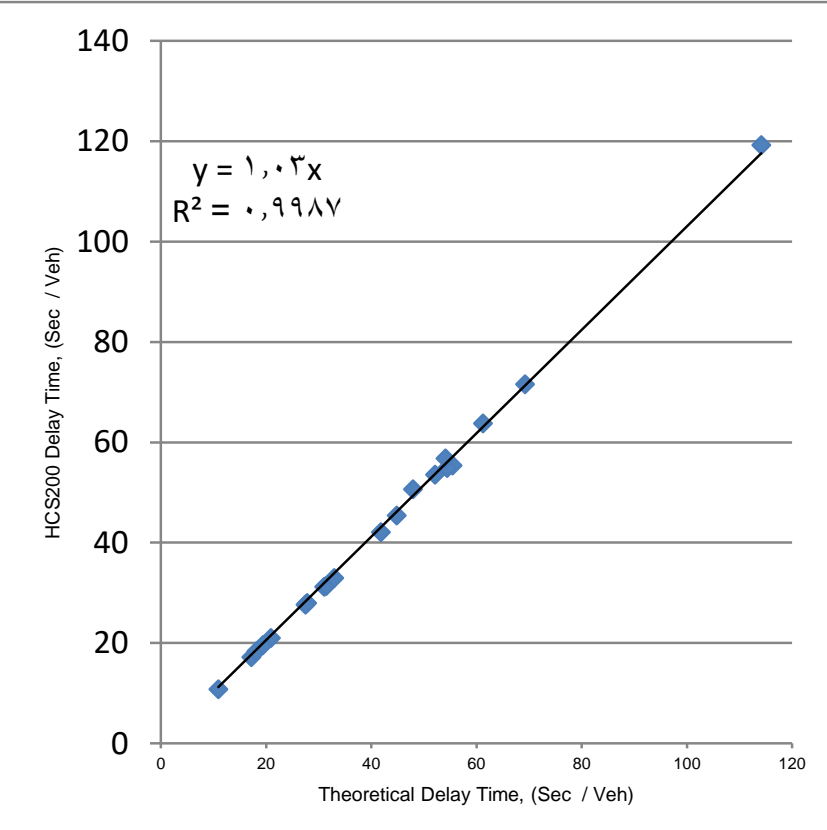

Figure (3): Relationship between Delay Times Calculated Theoretically and Delay Times Determined Using HCS2000 Software at Signalized Intersections

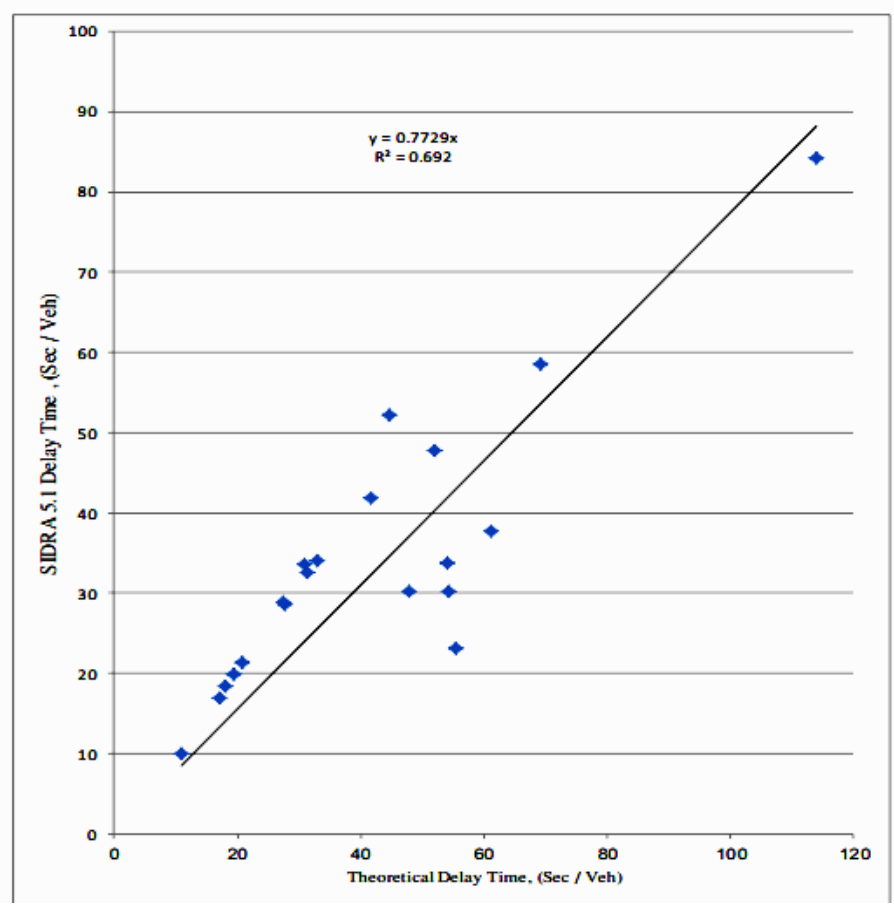

Figure (4): Relationship between Delay Times Calculated Theoretically and Delay Times Determined Using SIDRA 5.1 Software at Signalized Intersections.

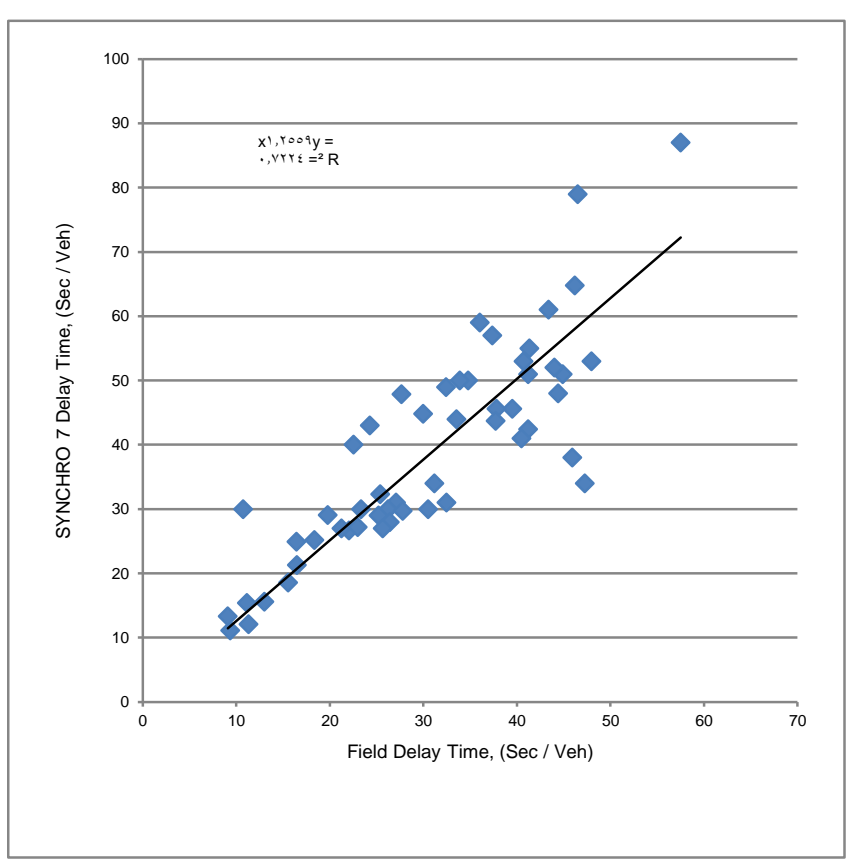

Figure (5): Relationship between Field Measurements Delay Times and Delay Times Determined Using SYNCHRO 7 Software. 


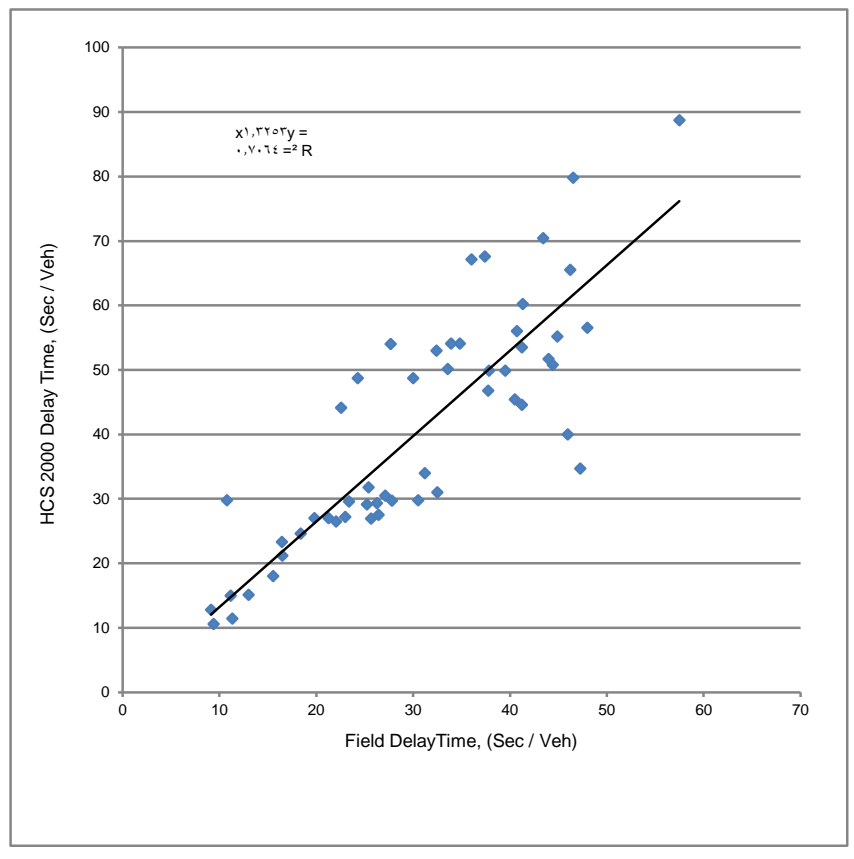

Figure (6): Relationship between Field Measurements Delay Times and Delay Times Determined Using HCS2000 Software.

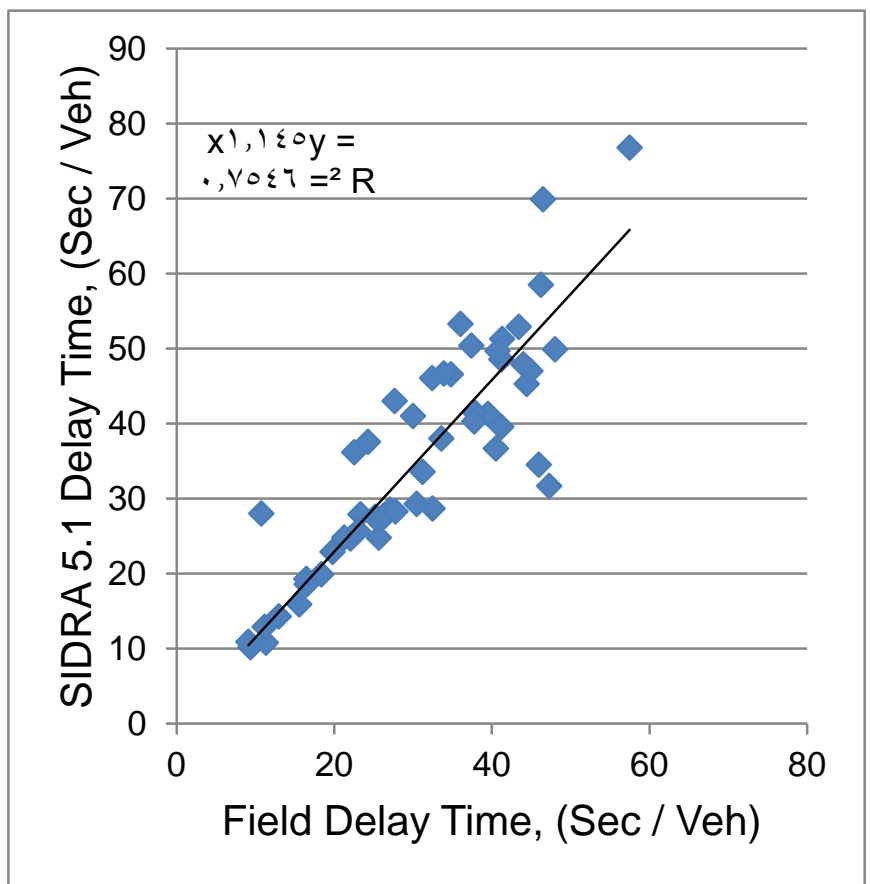

Figure (7): Relationship between Field Measurements Delay Times and DelaTimes Determined Using SIDRA Intersection 5.1 Software

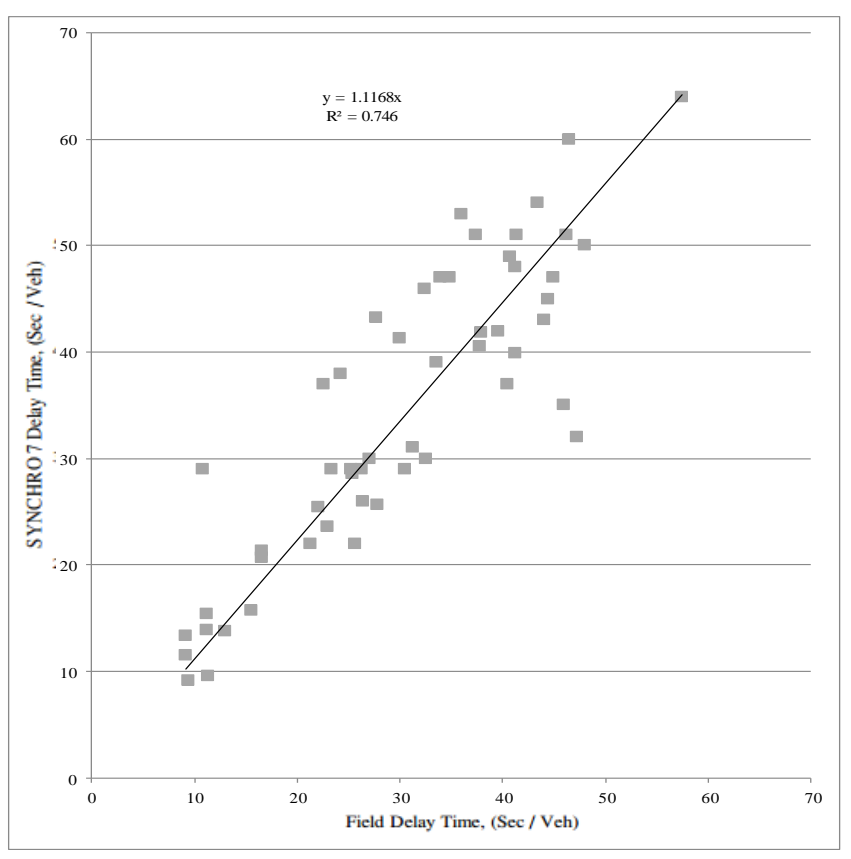

Figure (8): Relationship between Field Measurements Delay Times and Delay Times Determined Using SYNCHRO 7 at 1900 pcphgpl Base Saturation Flow

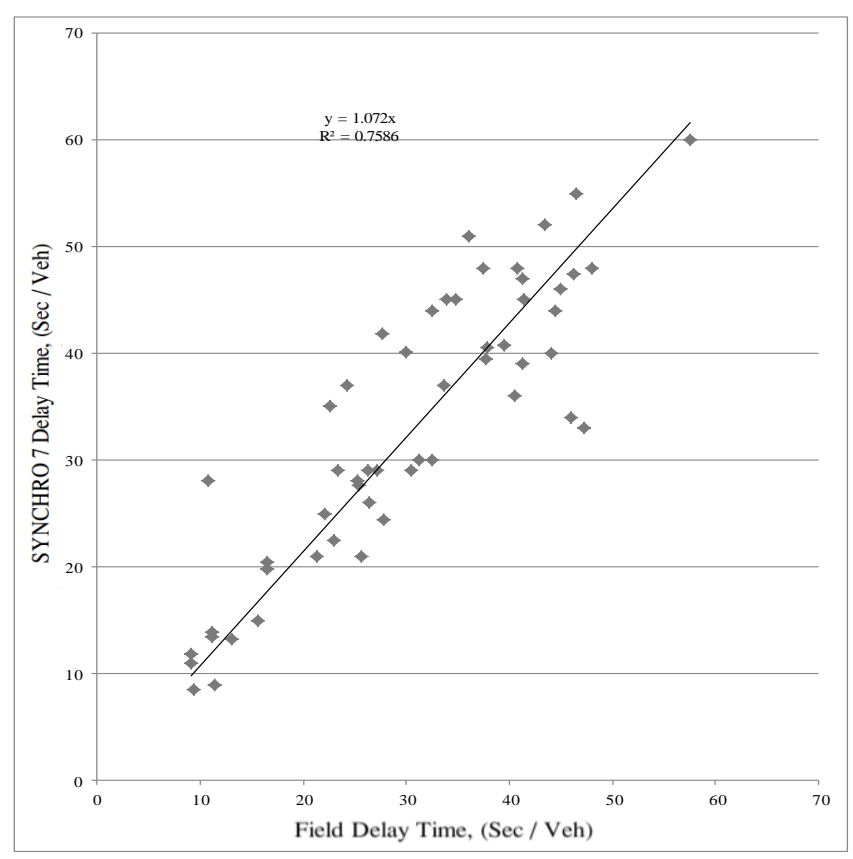

Figure (9): Relationship between Field Measurements Delay Times and Delay Times Determined Using SYNCHRO 7 at 2000 Pcphgpl Base Saturation Flow 


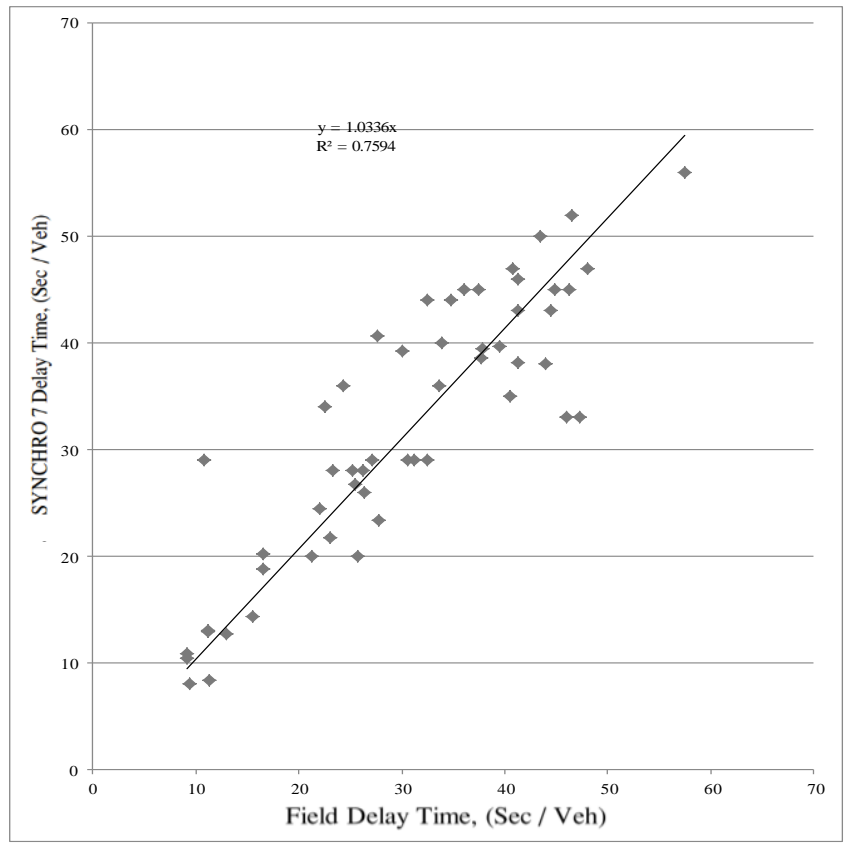

Figure (10): Relationship between Field Measurements Delay Times and Delay Times Determined Using SYNCHRO 7 at 2100 pcphgpl Base

$$
\text { Saturation Flow. }
$$

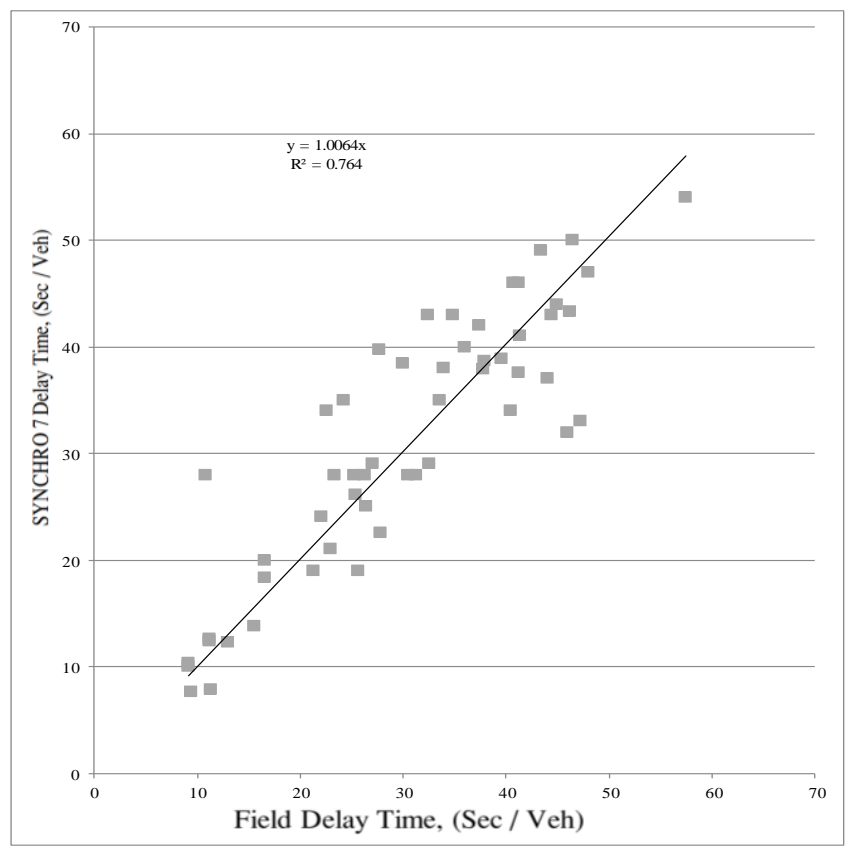

Figure (11): Relationship between Field Measurements Delay Times and Delay Times Determined Using SYNCHRO 7 Software at 2200 pcphgpl Base Saturation Flow.

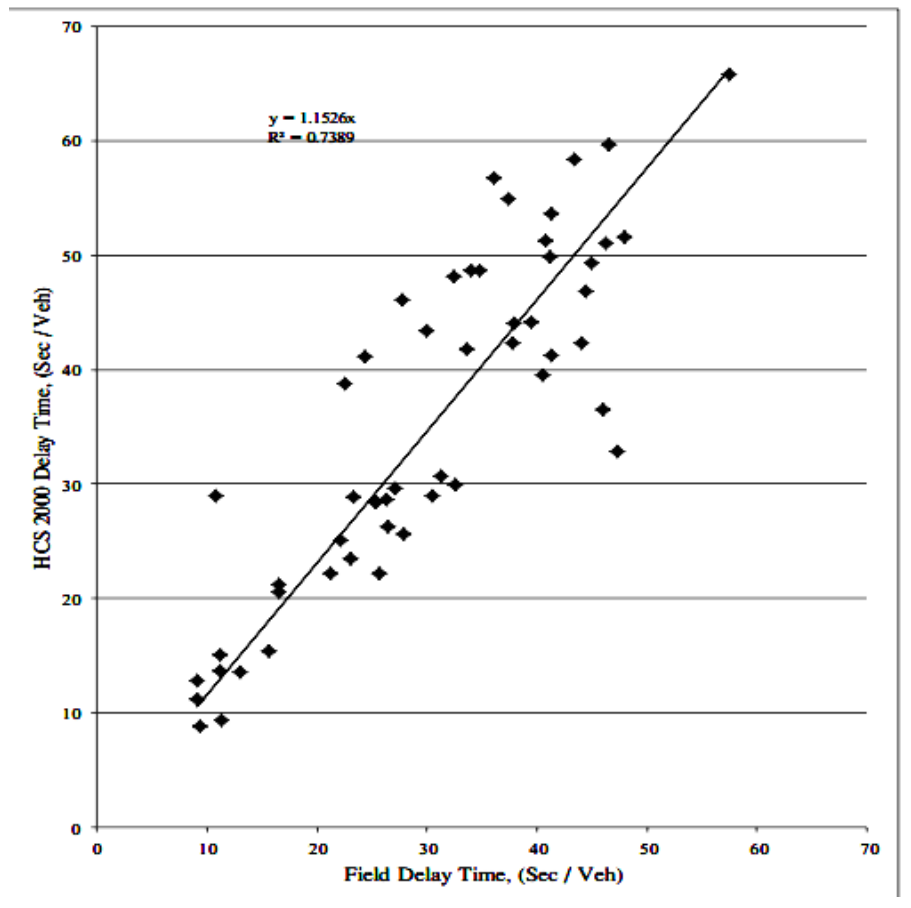

Figure (12): Relationship between Field Measurements Delay Times and Delay Times Determined Using HCS2000 at 1900 pcphgpl Base Saturation Flow.

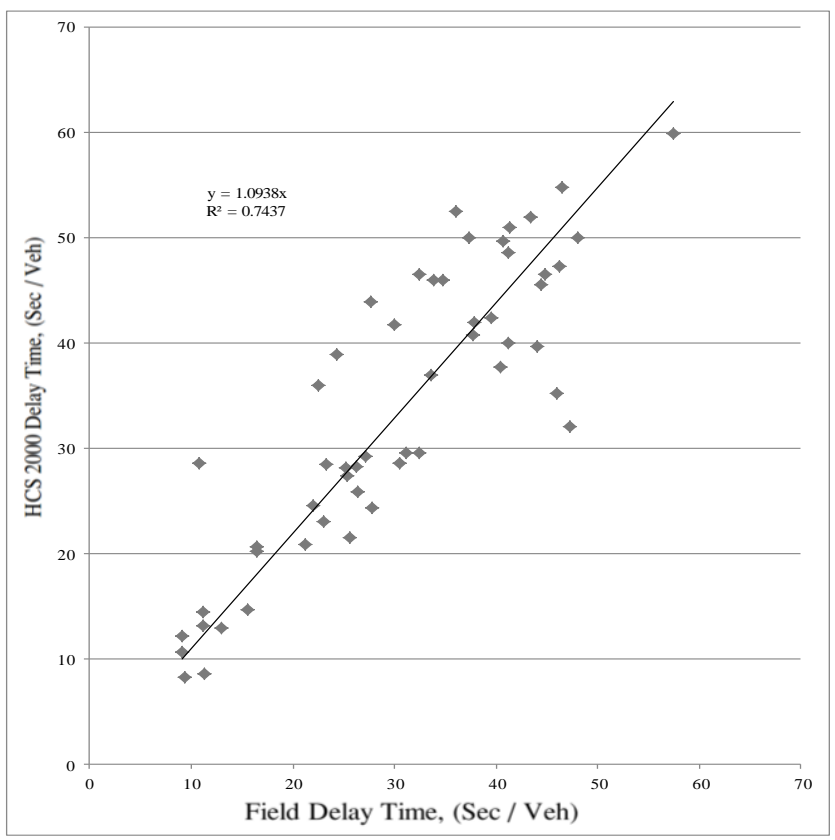

Figure (13): Relationship between Field Measurements Delay Times and Delay Times Determined Using HCS2000 Software at 2000 pcphgpl Base Saturation Flow. 


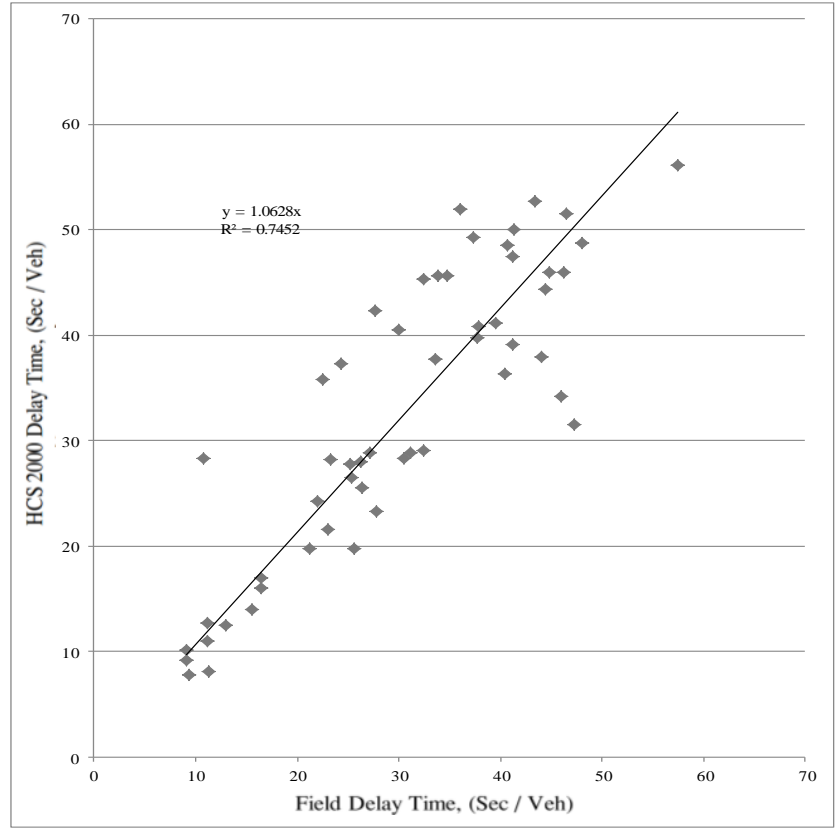

Figure (14): Relationship between Field Measurements Delay Times and Delay Times Determined Using HCS2000 at 2100 pcphgpl Base Saturation Flow.

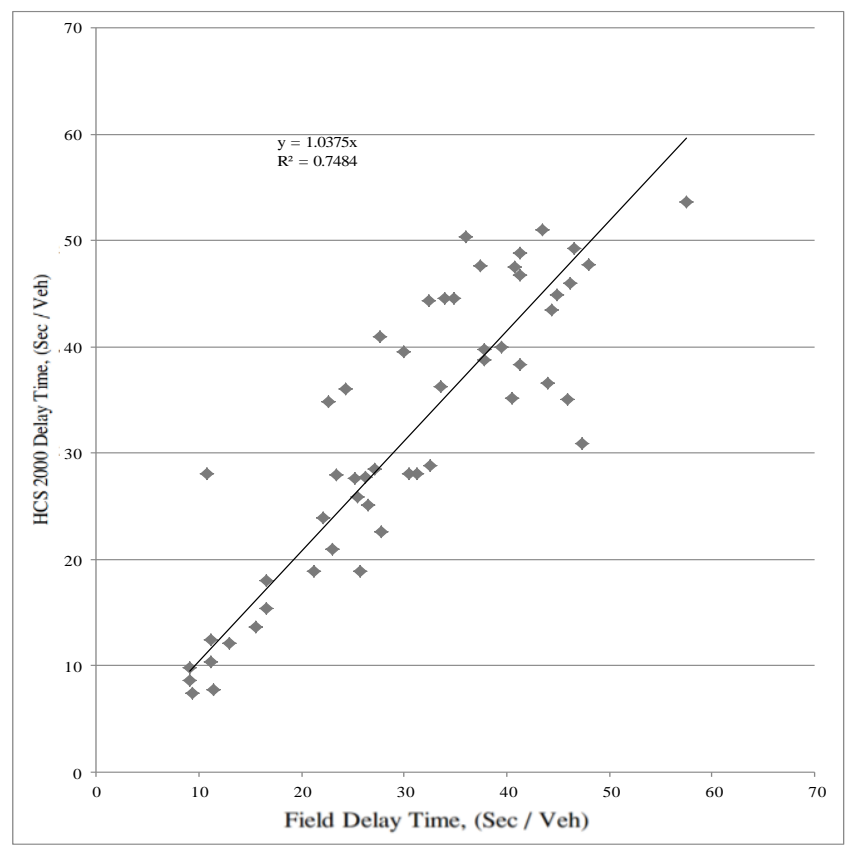

Figure (15): Relationship between Field Measurements Delay Times and Delay Times Determined Using HCS2000 at 2200 pcphgpl Base Saturation Flow.

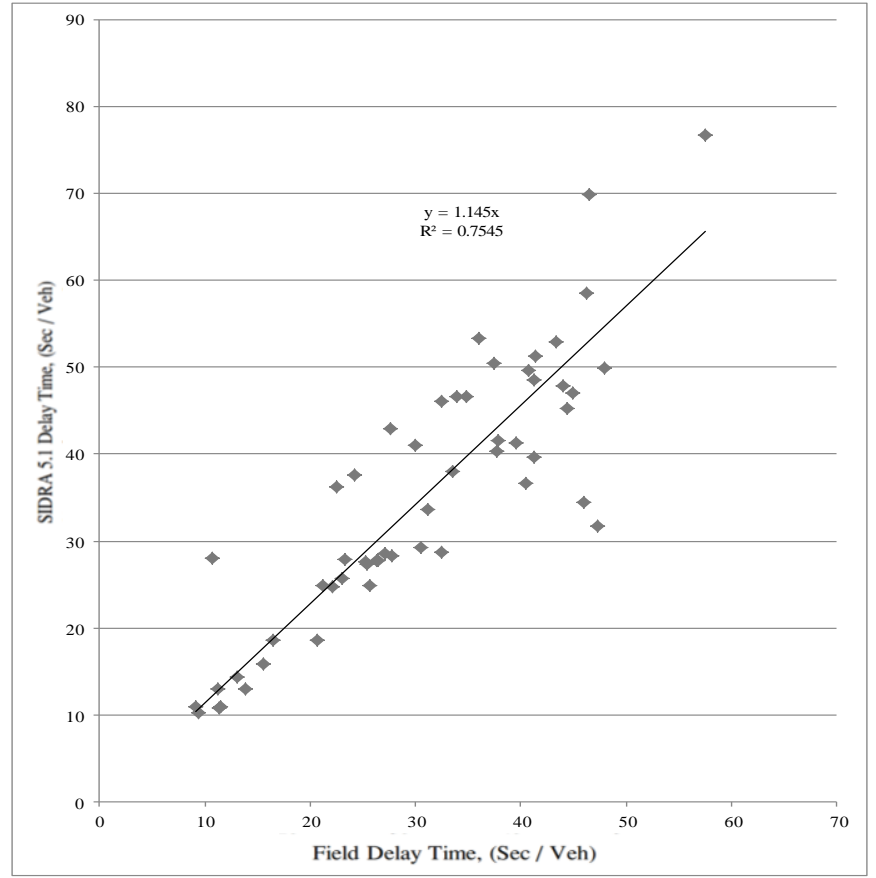

Figure (16): Relationship between Field Measurements Delay Times and Delay Times Determined Using SIDRA 5.1 at 1900 pcphgpl Base Saturation Flow.

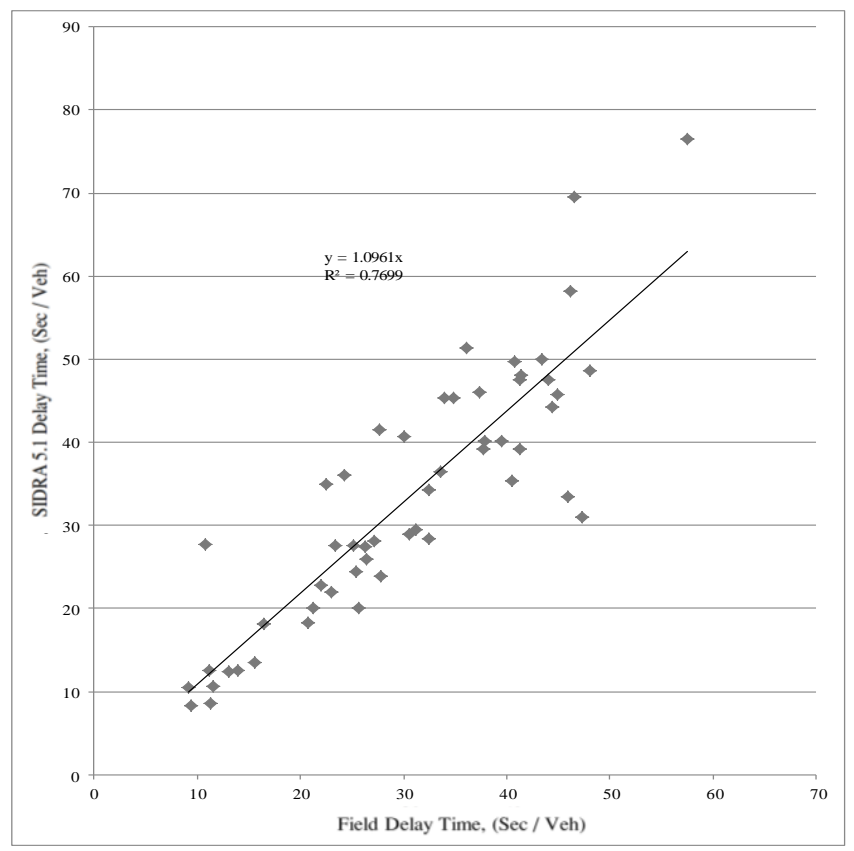

Figure (17): Relationship between Field Measurements Delay Times and Delay Times Determined Using SIDRA 5.1 at 2000 pcphgpl Base Saturation Flow 
C: 26 MANSOURA ENGINEERING JOURNAL, (MEJ), VOL. 41, ISSUE 2, JUNE 2016

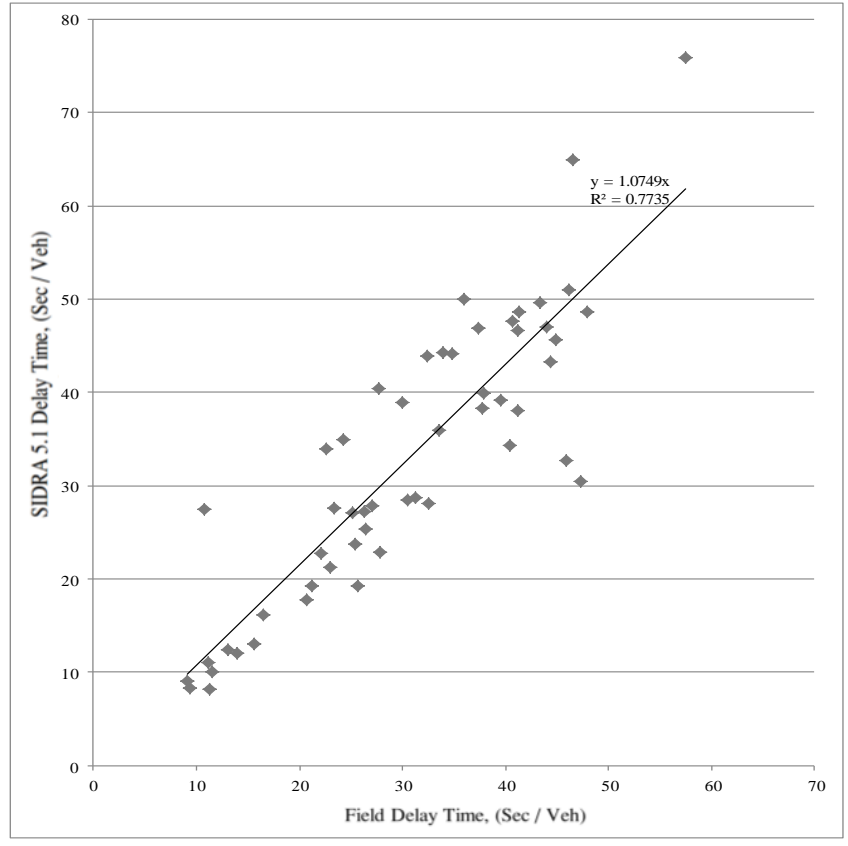

Figure (18): Relationship between Field Measurements Delay Times and Delay Times Determined Using SIDRA 5.1 at 2100 pcphgpl Base Saturation Flow.

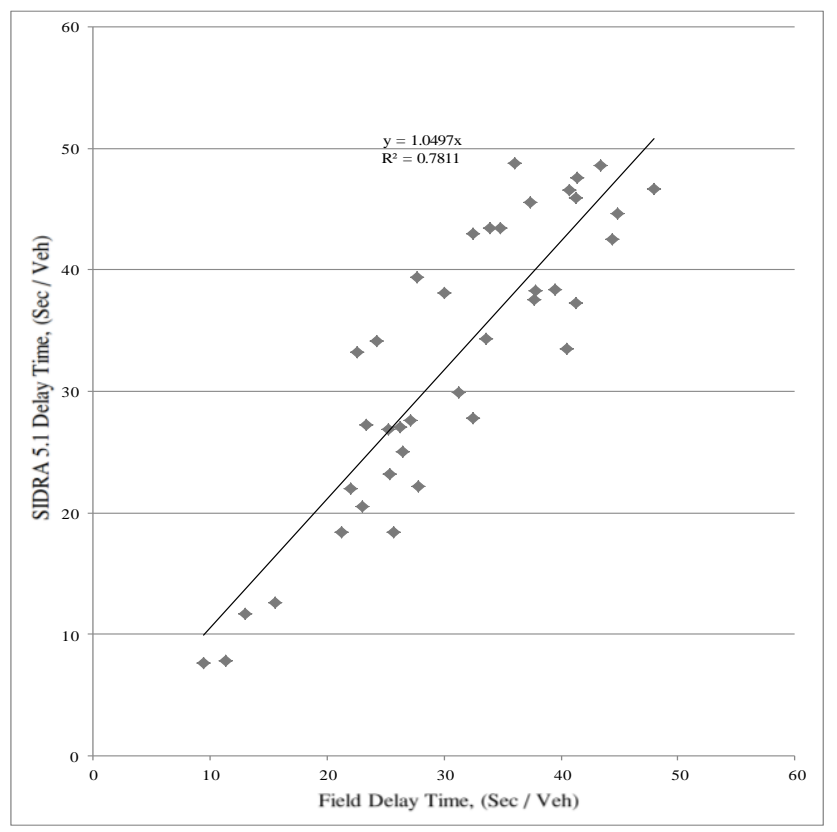

Figure (19): Relationship between Field Measurements Delay Times and Delay Times Determined Using SIDRA 5.1 at 2200 pcphgpl Base Saturation Flow.

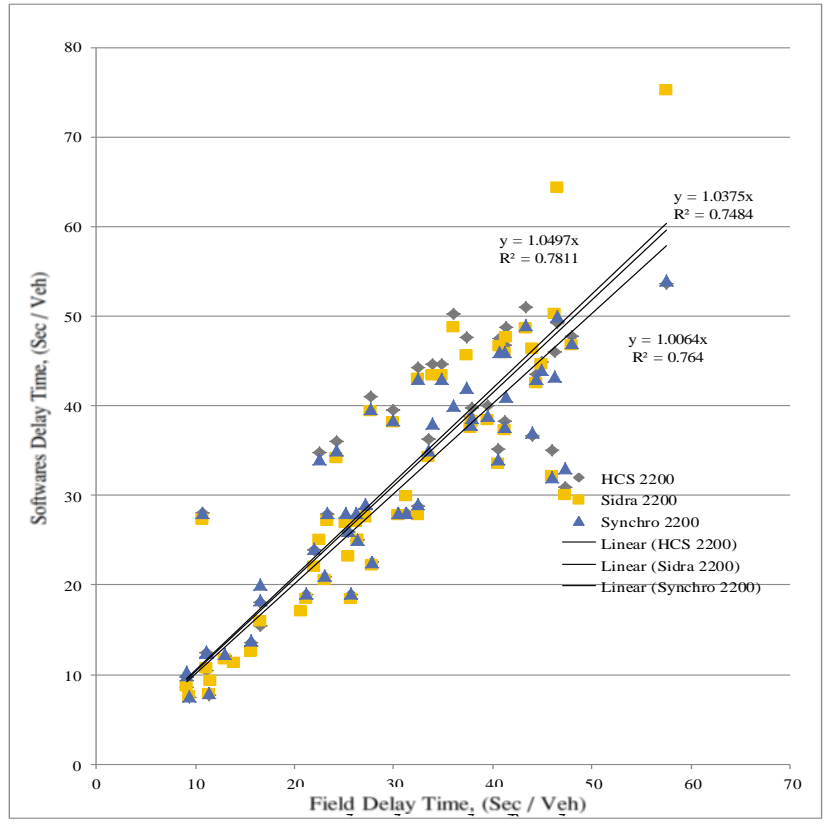

Figure (20): Relationships between Field Measurements Delay Times and Delay Times Determined Using Different Investigated Software Programs at 2200 pcphgpl Base Saturation Flow. 